\title{
Cellular and molecular changes to chondrocytes in an in vitro model of developmental dysplasia of the hip-an experimental model of DDH with swaddling position
}

\author{
BO NING $^{1 *}$, RUI JIN ${ }^{2}$, LIN WAN $^{3 *}$ and DAHUI WANG ${ }^{1}$ \\ ${ }^{1}$ Department of Pediatric Orthopedics, Children's Hospital of Fudan University, Shanghai 201102; \\ ${ }^{2}$ Department of Pediatric Orthopedics, Children's Hospital of Anhui Medical University, Hefei, Anhui 230051; \\ ${ }^{3}$ Department of Cardiothoracic Surgery, Shanghai Children's Hospital, Shanghai 230041, P.R. China
}

Received March 25, 2018; Accepted July 19, 2018

DOI: $10.3892 / \mathrm{mmr} .2018 .9384$

\begin{abstract}
The aim of the present study was to assess the cellular and molecular changes to chondrocytes in a developmental dysplasia of the hip (DDH) model and to investigate the early metabolism of chondrocytes in DDH. Neonatal Wistar rats were used for the DDH model with swaddling position. Primary cultures of chondrocytes were prepared at serial interval stages $(2,4,6$ and 8 weeks) to investigate cellular proliferation. The expression of collagen II and aggrecan mRNA was detected to assess the anabolic ability of chondrocytes. The expression of matrix metallopeptidase (MMP)-13 and ADAM metallopeptidase with thrombospondin type 1 motif 5 (ADAMTS-5) mRNA was measured to investigate the degradation of collagen II and aggrecan, respectively. Morphological changes were observed in coronal dissection samples after the removal of fixation. Primary chondrocytes at serial intervals were assessed using a Cell Counting Kit- 8 assay and the results revealed that DDH chondrocytes had more proliferative activity. The expression of collagen II mRNA was upregulated at 2 weeks and was more sensitive to mechanical loading compared with aggrecan. Similar changes occurred at 6 weeks. Furthermore, MMP-13 and ADAMTS-5 mRNA expression levels were upregulated at 2 weeks. It was also demonstrated that DDH chondrocytes exhibited high proliferative activity at the early stages and degeneration later.
\end{abstract}

Correspondence to: Dr Dahui Wang, Department of Pediatric Orthopedics, Children's Hospital of Fudan University, 399 Wanyuan Road, Shanghai 201102, P.R. China

E-mail: dr_dahuiwang@126.com

*Contributed equally

Key words: developmental dysplasia of the hip, animal model, cell proliferative, cartilage

\section{Introduction}

Developmental dysplasia of the hip (DDH) is one of the most common malformations affecting the lower extremities in children. If a stable and concentric reduction is maintained after close reduction, the acetabulum has the potential to remodel and resume normal growth and development. Furthermore, if younger patients are at the onset of treatment, this results in a greater potential for acetabular remodeling (1-5). The use of magnetic resonance imaging has resulted in enhanced consideration of the importance of acetabular cartilage in hip remodeling $(6,7)$. Chondrocytes, one of the important components of cartilage, serve a critical role in maintaining the function and biological features of cartilage. However, it is difficult to obtain hip cartilage from DDH patients due to ethical constraints. Consequently, the function and pathophysiology of chondrocytes in the hips of patients with DDH cannot be in investigated in vivo. In addition, it is not possible to obtain cultured human chondrocytes from patients with DDH. Due to these constraints, animal models are used to improve our understanding of chondrocytes in DDH in vivo. A rat model of unilateral DDH was established by Sijbrandij (8), and since then various experimental animals have been used to show that remodeling of the acetabulum is possible after the removal of fixation $(9,10)$. However, previous studies mainly focused on the morphological and histological alterations of abnormal hips and the corresponding cartilage (11-13). Furthermore, since the swaddle position in infants is considered to be an important risk for the development of DDH, the model designed by the principle is thought to be an accurate model of cartilage in human DDH and may be suitable for further investigations (14). The authors previously developed a successful neonatal rat model of DDH corresponding to the swaddling position of the hip and early cartilage degeneration in DDH (10).

The aim of the present study was to assess the features of chondrocytes in DDH cartilage via primary cell culture in vitro. DDH models of neonatal Wistar rats were prepared in the present study and serial sections of hip cartilage were isolated and incubated primarily to investigate the cellular characteristics after the removal of fixation. 


\section{Materials and methods}

Experimental animal models. All experimental protocols were approved by the Animal Ethical Committee of Fudan University (Shanghai, China). A total of 80 male specific pathogen free neonatal Wistar rats $(\sim 5 \mathrm{~g})$ were purchased from the Animal Research Institute of Medical College of Fudan University. Feeding environment was as follows: Temperature, $21-26^{\circ} \mathrm{C}$, relative humidity $45-65 \%$, ventilation for 8-12 times/h, 12-h light/dark cycle. The rats were fed with sterile pure water and adequate feed (HFK bio-technology, Beijing, China). Rats in the experimental DDH group ( $\mathrm{n}=40$ ) were immobilized, with the hip and knee fixed in an extended position with medical tapes for 10 days as described in our previous study $(10,12)$. Following the removal of the fixation, rats were allowed to move freely in their cage for $2,4,6$ or 8 weeks. Rats were sacrificed and the hips were isolated for macro-morphological examination and primary cell culture of the articular cartilage. Rats in the control group $(n=40)$ were allowed to move freely throughout the study period.

Coronal histology and morphometry. Hips were isolated and fixed in $4 \%$ paraformaldehyde for $24 \mathrm{~h}$ in room temperature and decalcified with 10\% EDTA, following which they were dissected through the longitudinal line from the ilium to the ischium of acetabulum. An abnormal association between the acetabulum and femoral head was observed in the experimental DDH group. The largest coronal that was selected from sections $(5-\mu \mathrm{m})$ and acetabular index was measured. The acetabular depth ratio (ADR=depth/width $\times 100 \%)$ was measured to assess changes in the acetabulum (Fig. 1A). The acetabular index (AI) was defined as the ratio of depth: Width measured using the reference line presented in Fig. 1A. The long line is identified as the width diameter of the longitudinal acetabulum from the upper edge to the distal border, excluding the rim of labrum. The short line is the perpendicular line to the width diameter of the acetabulum, and the curved line represents the acetabular shape following the removal of fixation.

Primary cell culture protocol. Primary cell culture was performed using the modified Manning method (15). Cartilage was obtained from the hips under sterile conditions, minced into $1 \mathrm{~mm}^{3}$ pieces with scissors and digested using $0.25 \%$ Trypsin-EDTA and 2\% collagenase II (Invitrogen; Thermo Fisher Scientific, Inc., Waltham, MA, USA). Tissues were passed through a $0.25 \mu \mathrm{m}$ molecular filter and the cell suspension was centrifuged at $240 \mathrm{xg}$ for $5 \mathrm{~min}$ in room temperature. The resulting pellet was resuspended in fresh Dulbecco's modified Eagle's medium (DMEM; Sigma-Aldrich; Merck KGaA, Darmstadt, Germany), supplemented with $10 \%$ fetal bovine serum (Sigma-Aldrich; Merck KGaA), $5 \mu \mathrm{g} / \mathrm{ml}$ penicillin and $5 \mu \mathrm{g} / \mathrm{ml}$ streptomycin. The final cell density was $5 \times 10^{6}$ cells $/ \mathrm{ml}$ and the suspension was incubated at $37^{\circ} \mathrm{C}$ in an atmosphere containing $5 \% \mathrm{CO}_{2}$ overnight, to allow primary chondrocytes to adhere. The medium was replaced with fresh DMEM every two days. Cells were then identified using collagen II immunofluorescence staining.

Chondrocyte identification using collagen II immunofluorescence. The medium was discarded and cells were rinsed three times with PBS for 5 min. Cell samples were fixed with $4 \%$ paraformaldehyde for $30 \mathrm{~min}$ at room temperature, following which, they were blocked in $0.2 \%$ Triton X-100 (PBST; Sigma-Aldrich; Merck KGaA) mixed with goat serum (Sigma-Aldrich; Merck KGaA) for $30 \mathrm{~min}$ at $37^{\circ} \mathrm{C}$. Cells were immunoblotted and incubated overnight at $4^{\circ} \mathrm{C}$ with primary antibodies against collagen II (cat. no. ab34712; Abcam, Cambridge, UK; 1:100). The specimens were incubated for $1 \mathrm{~h}$ with secondary antibodies goat anti-rabbit IgG Alexa Fluor ${ }^{\circledR}$ 488 (cat. no. ab150077 Abcam; 1:200) at room temperature in the dark. Finally, specimens were stained with DAPI at room temperature and then viewed under an inverted fluorescence microscope (excitation wavelength $488 \mathrm{~nm}$, magnification 200X) and images were captured. Collagen II staining at different time (2, 4, 6 and 8 weeks) was assessed and the number of cells was counted using Image Plus Pro 6.0 (Media Cybernetics, Inc., Rockville, MD, USA).

Cell proliferation kinetics and growth curve. Primary cells were seeded in a 96-well plate at a density of 4,000 cells $/ 200 \mu 1$ and the number of chondrocytes was assessed using a Cell Counting Kit (CCK)-8 (DJDB4000X; Dojindo Molecular Technologies, Inc., Kumamoto, Japan) each day for 1 week. The optical density (OD) value of CCK- 8 absorbance was measured at a wavelength of $450 \mathrm{~nm}$ using an ELISA reader and used to construct the growth curve.

Cell cycle analysis. Samples were centrifuged at $300 \mathrm{x}$ g for 3 min at $4^{\circ} \mathrm{C}$, then collected and rinsed with cold PBS. Cells were then fixed with $70 \%$ cold ethanol at $4^{\circ} \mathrm{C}$ overnight, then the cell were treated with Cell Cycle Detection kit (KGA512, Nanjing KeyGen Biotech Co., Ltd., Nanjing, China). Cell cycle analysis was performed using flow cytometry equipment (FACSAria II; BD Biosciences, Franklin Lakes, NJ, USA) and the data were collected using FlowJo analysis software (FlowJo-10.5.0; Flowjo LLC, Ashland, OR, USA). The number of cells in each phase of the cell cycle was recorded and the proportion of cells in S-phase was taken to be representative of proliferative activity.

mRNA expression levels of collagen II, aggrecan, matrix metallopeptidase (MMP)-13 and ADAM metallopeptidase with thrombospondin type 1 motif 5 (ADAMTS-5). Total RNA was extracted from the monolayer confluent chondrocytes using TRIzol ${ }^{\circledR}$ (Invitrogen; Thermo Fisher Scientific, Inc.) and the purity and integrity were assayed using spectrophotometry and $10 \%$ agarose-gel electrophoresis respectively. The OD values of these mRNAs were detected between 1.8 and 2.0. A total of $1 \mu \mathrm{g}$ RNA was transcribed to produce cDNA using a ReverTra Ace qPCR RT kit (Toyobo, Life Science, Osaka, Japan) according to the manufacturer's protocol. The yield was quantified spectrophotometrically.

Reverse transcription-quantitative polymerase chain reaction (RT-qPCR) (Denaturing $94^{\circ} \mathrm{C}, 60 \mathrm{sec} ; 40$ cycles (Denaturing $94^{\circ} \mathrm{C}, 10 \mathrm{sec}$; annealing and extension $60^{\circ} \mathrm{C}$, $30 \mathrm{sec}$ ) was performed using $5 \mu \mathrm{l}$ cDNA (100 ng), $2 \mu \mathrm{l}$ each primer $(10 \mu \mathrm{M}), 25 \mu \mathrm{l}$ SYBR Green Real-time PCR Master Mix (Toyobo, Life Science) and $16 \mu 1$ water to give a total volume of $50 \mu \mathrm{l}$. The RT-qPCR was programmed to an initial step of $10 \mathrm{~min}$ at $95^{\circ} \mathrm{C}$ for polymerase activity, followed by 

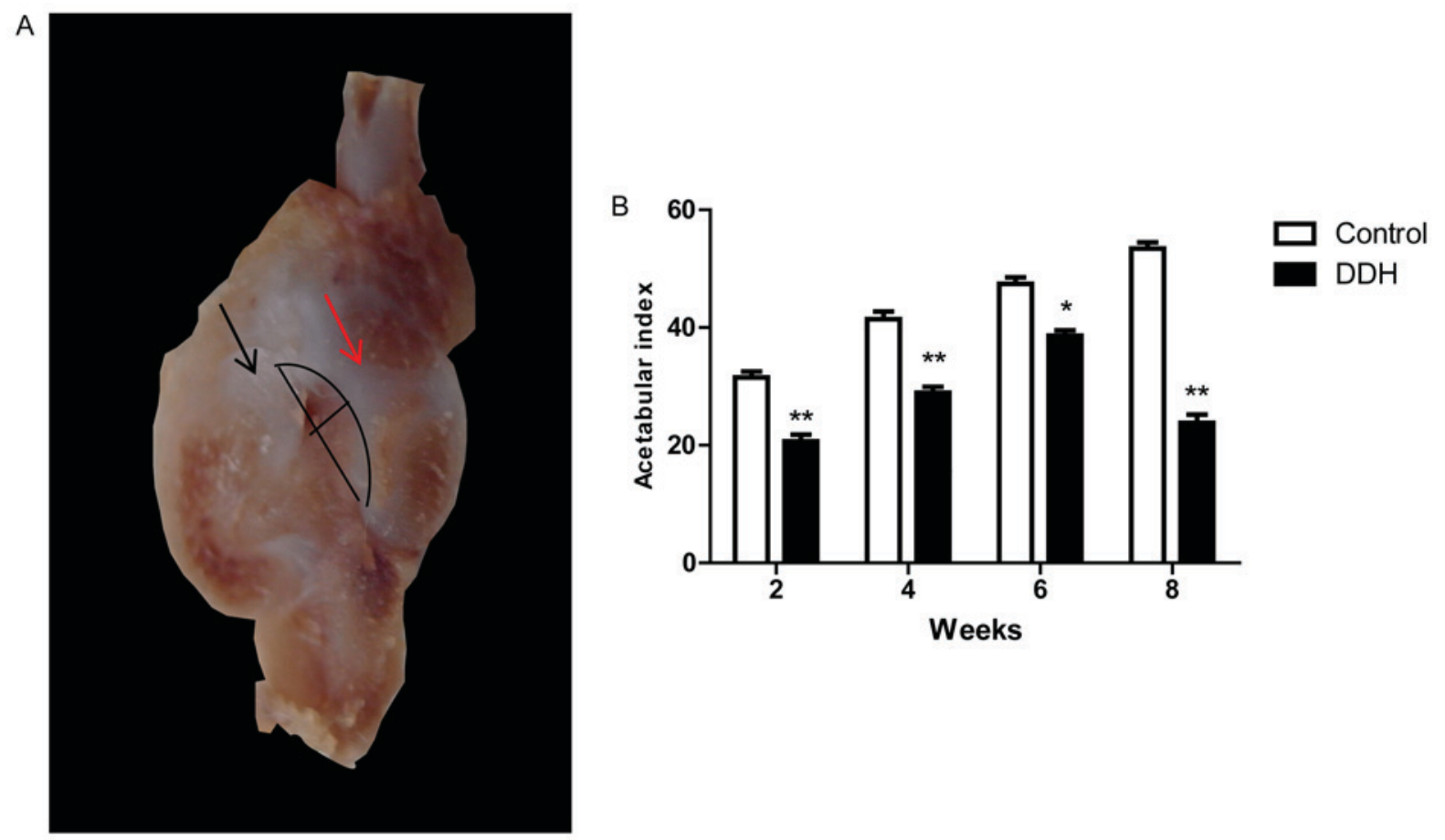

Figure 1. Model and morphometry of DDH. (A) Coronal dissection presenting the morphology of the hip in a DDH model. Black arrow indicates the acetabulum and red arrow indicates the femoral head. The AI was defined as the ratio of depth to width, and was measured using the reference line shown in the figure. The width of the longitudinal acetabulum from the upper edge to the distal border excluded the rim of labrum. The line perpendicular to the width of the acetabulum and the curve line represent the acetabular shape after the removal of fixation. (B) Changes to the AI at serial intervals. ${ }^{*} \mathrm{P}<0.05$ and ${ }^{* *} \mathrm{P}<0.001$ vs. control. AI, acetabular index; DDH, developmental dysplasia of the hip.

40 cycles of $15 \mathrm{sec}$ denaturation at $95^{\circ} \mathrm{C}, 15 \mathrm{sec}$ annealing at $60^{\circ} \mathrm{C}$, and $45 \mathrm{sec}$ extension at $72^{\circ} \mathrm{C}$. The expression levels of MMP-13, Collagen 2a1, ADAMTS-4 and ADAMTS-5 were normalized to $\beta$-actin. All primers used are listed in Table I. The results were quantified using the $2^{-\Delta \Delta C q}$ method (16).

Statistical analysis. Data are expressed as the mean \pm standard error of the mean. Statistical significance was determined using one-way analysis of variance and paired t-tests. The bonferroni method was used for post hoc tests. Data were analyzed using SPSS software, version 16.0 (SPSS, Inc., Chicago, IL, USA). Each experiment was repeated three times. $\mathrm{P}<0.05$ was considered to indicate a statistically significant difference.

\section{Results}

Model and morphometry. Gross observations of the coronal dissection morphology of the hip are presented in Fig. 1A. A distinct DDH model was identified in the experimental DDH group, with dislocational hips observed (Fig. 1A). The acetabular index (AI) was significantly lower in the DDH rats compared with the control group at all time points $(\mathrm{P}<0.001$; Fig. 1B).

Immunofluorescence staining of collagen II and evaluation of cell proliferation. The expression of collagen II was used to identify chondrocytes. No apparent differences in cell morphology were observed between the control and experimental DDH groups (Fig. 2A). Cell morphology varied, with cobblestone, ellipse and polygonal shapes observed after the primary cells had adhered (Fig. 2A). In addition, cell proliferation was assessed by counting cells following staining with DAPI (Fig. 2B). The results revealed increased proliferation in the experimental DDH group compared with the control group at all time points $(\mathrm{P}<0.001)$.

Cell growth curve. At 2 weeks, the growth curve for the experimental DDH group began to enter the linear phase, the slope of which was greater than the control group, which indicated an increased trend of proliferation (Fig. 3A). However, no significant differences in the linear phase slope were observed between groups at 4 weeks. This suggested that proliferation in the experimental DDH group slowed over time (Fig. 3B). Furthermore, at week 6 the slope of the linear phase was reversed (Fig. 3C); this was maintained until 8 weeks with the experimental DDH group exhibiting a decreased slope (Fig. 3D).

Number of cells in $S$ phase increases during DDH. Flow cytometry was performed to measure the proportion of cells in each phase of the cell cycle at different time points (Fig. 4). $\mathrm{S}$-phase is when DNA synthesis occurs, and is therefore reflective of cell proliferation. At all time points, a greater number of cells in the experimental DDH group were in S-phase compared with the control group $(\mathrm{P}<0.001$; Fig. 4A). Following the 2 week period, a gradual increase in the proportion of cells in S-phase was observed in the experimental DDH group over time (Fig. 4C). A reduced number of cells were observed in G1-phase in the experimental DDH group compared with the control, particularly at 2 and 8 weeks (Fig. 4B). The number of experimental DDH cells in G2-phase was also lower compared with the control group, particularly at 2 weeks (Fig. 4D). A 
Table I. Primers used for amplification of target genes and $\beta$-actin.

\begin{tabular}{ll}
\hline Gene & \multicolumn{1}{c}{ Sequence (5'-3) } \\
\hline Collagen II & \\
F & ACGCTCAAGTCGCTGAACAA \\
R & TCAATCCAGTAGTCTCCGCTCT \\
Aggrecan & \\
F & TCCAAACCAACCCGACAAT \\
R & TCTCATAGCGATCTTTCTTCTGC \\
MMP-13 & \\
F & TACGAGCATCCATCCCGAGACC \\
R & AACCGCAGCACTGAGCCTTTTC \\
ADAMTS-5 & \\
F & GGCTGTGGTGTGCTGTG \\
R & CTGGTCTTTGGCTTTGAAC \\
$\beta$-actin & \\
F & GGAGATTACTGCCCTGGCTCCTA \\
R & GACTCATCGTACTCCTGCTTGCTG \\
\hline
\end{tabular}

F, forward; R, reverse; MMP-13, matrix metallopeptidase; ADAMTS-5, ADAM metallopeptidase with thrombospondin type 1 motif 5 .

significant increase in experimental DDH cells in the S-phase was observed at 2 and 8 weeks (Fig. 4C).

mRNA expression levels of collagen II, aggrecan, MMP-13 and ADAMTS-5 are varied at different time-points in $D D H$. The mRNA expression levels were investigated using RT-qPCR (Fig. 5). Collagen II mRNA expression in the experimental DDH group was upregulated at 2 weeks after the removal of fixation, and downregulated at 4 weeks. However, a significant reversal was present at 6 weeks followed by a downregulation again at 8 weeks (Fig. 5A). The expression of MMP-13 mRNA was overexpressed in DDH cells compared with the control group at 2 weeks, decreased at 4 weeks and then this expression gradually increased during the following weeks (Fig. 5C). Aggrecan expression levels were significantly different between the experimental DDH and control groups at weeks 4 and 8; however, no significant differences were observed at weeks 2 and 6 (Fig. 5B). Conversely ADAMTS-5 mRNA in the DDH group was significantly different at weeks 2, 6 and 8 compared with the control (Fig. 5D).

\section{Discussion}

The DDH model of neonatal rats shows that the swaddling position is a mechanical risk factor that plays an important role in the pathogenesis of DDH. Previous studies of DDH models have revealed that the hip may be remodeled after the removal of fixation, which resembles close reduction treatment in human infants with DDH (17). Furthermore, Yamamoto (2) reported that shorter fixation duration gave better results. It has also been reported that the $\mathrm{DDH}$ is able to be completely reversed to prevent degeneration (18). Nevertheless, the results of the present study were not consistent with previous reports due to the failure to achieve close reduction; although remodeling of the macro-morphology was observed in the early stage, degeneration increased irreversibly with skeletal maturity $(10,12)$. However, changes may be associated with cartilage content and resulting differences in mechanical features at different ages. In addition, the maintenance of subluxation resulted in no complete reduction, as the long duration of immobilization made the cartilage suffer from more abnormal weight-bearing. These kinds of changes leading to cartilage degeneration can be observed in clinical DDH X rays.

The histological and gross observation results revealed that remodeling occurred with proliferation at an early stage. Few studies focus on cellular proliferation of the chondrocytes, no matter whether or not the cells are loaded with abnormal mechanical forces. It is therefore important to investigate the proliferative ability at a cellular level (19). Chondrocytes in patients with DDH suffer from a variety of stresses, including shear, compression and tension loading, and so it is difficult to analyze the relationship between cell proliferation and mechanical loading. A number of studies have investigated the association between simple stress in vitro and cell proliferation and demonstrated that proliferation is associated with the type, intensity and mode of stress (20-25). In addition, a novel method for assessing articular cartilage chondrocytes in vivo has been described (26). However, the effects of different types of stress loading on the cartilage cannot be examined in vivo in a rat model, as the hip volume is too small. In the present study, cell proliferation was assessed using cell cycle analysis, and CCK-8 assays.

Cell cycle progression is the predominant means of regulating cell proliferation and differentiation, and so increasing our understanding of cell cycle progression in DDH chondrocytes may be beneficial. Changes in the number of cells in S-phase at 2 weeks may be due to an increase in proliferative ability following the transient removal of compress loading. The changes at weeks 4 and 6 may occur as a result of sustained complex loading due to mobilization, and accumulation of the proliferative cells may be a compensational reaction to maintain cartilage function. However, it is unclear why at 8 weeks cell numbers decreased while the proportion of cells in S-phase increased. Although an increase in S-phase cells indicated early proliferative activity, the proportion of cells in G0/G1-phase was significantly lower in the experimental DDH group compared with the control group, suggesting that proliferation and differentiation may be elevated in DDH chondrocytes. Nevertheless, the proliferation index regarding the S-phase accounted for the cell proliferative ability at the actual time points, while CCK- 8 results indicated proliferative kinetics according to the slope of the growth curve. The results revealed that proliferation occurred faster soon after the removal of fixation in DDH chondrocytes.

The relationship between cartilage remodeling and cell proliferation has recently been reported by assessing the spatial reorganization of superficial chondrocytes in the early stages of osteoarthritis (27). Furthermore, a clear association has been reported between cell proliferation and ECM metabolism (28). Further investigation is required to discover further details regarding changes in ECM metabolism in DDH chondrocytes in response to changes in loading. 
A

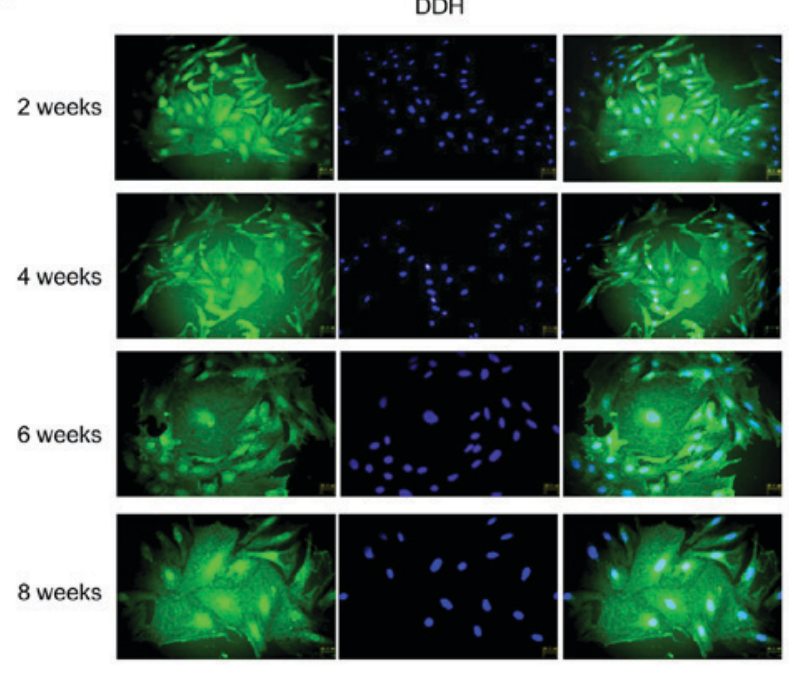

Control
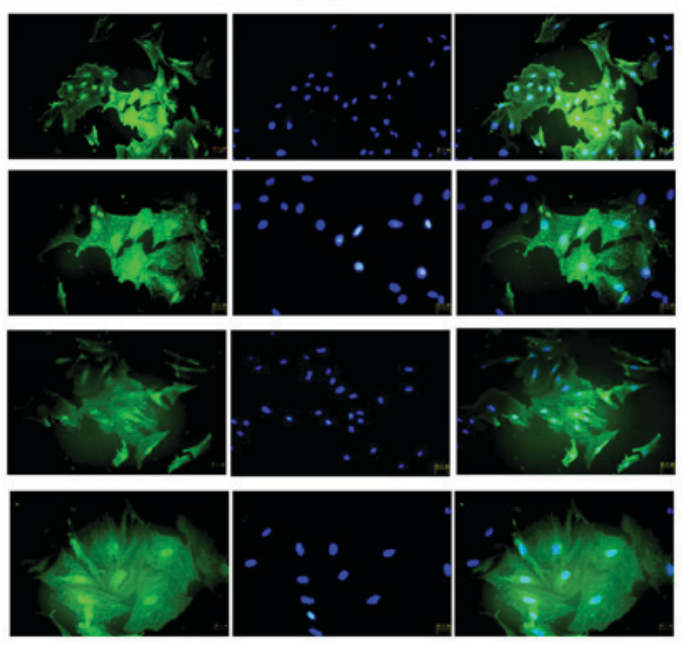

B

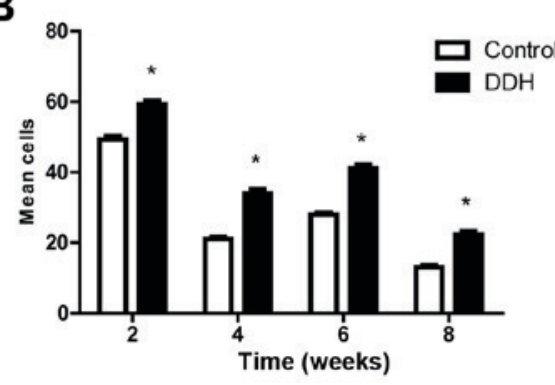

Figure 2. Immunofluorescence staining of collagen II and evaluation of cell proliferation during DDH degeneration. (A) Immunostaining of collagen II at different time points. Collagen II was primarily expressed in the nucleus at all time points. Hypertrophic chondrocytes were observed in the experimental DDH group at week 6, indicating that chondrocytes became pre-hypertrophic and were prepared for maturity following degeneration of the chondrocytes. Magnification, $x 200$ and scale bar $=1 \mu \mathrm{m}$. (B) Cell proliferation. At 2 weeks, the cell number increased with the transient removal of fixation, and differences were observed between groups at all time points. Increased proliferation was observed in the experimental DDH group compared with the control groups. The number of cells in the experimental DDH group decreased at 8 weeks compared with 6 weeks, but remained increased compared with than in 8 -week control group. ${ }^{\mathrm{P}}<0.05$ vs. control. DDH, developmental dysplasia of the hip.

Collagen II and aggrecan provide the cartilage with tensile and compressive strength by forming a meshwork of collagen II in which the interstices are filled with aggrecans $(29,30)$. MMPs and ADAMTSs secreted by chondrocytes are the two main groups of proteases in the ECM that mediate the degradation of collagen II and aggrecan. mRNA was extracted from primary cells and the expression of Col2al and aggrecan were assessed along with MMP-13 and ADAMTS-5 expression.

Homeostasis of the cellular environment is important for the function of the cartilage, maintaining a balance between the structural components and their proteolytic enzymes in response to dynamic loading (31). If chondrocyte metabolism is disrupted due to abnormal mechanical stresses and degradation of the ECM, the chondrocytes will initiate a compensational mechanism to counteract the inappropriate mechanical loading. Studies have revealed that cartilage regeneration and degeneration are dependent on the duration, quality and strength of abnormal loading $(32,33)$. However, results have indicated that the metabolism of collagen in response to abnormal loading is different to that of aggrecan (20). As such, the upregulation of collagen II independent of aggrecan is considered to be a marker for early degeneration in DDH experiments as well as early osteoarthritis (11,34-36). In the present study, no significant differences in expression were observed between collagen
II and aggrecan at 2 weeks. However, both were upregulated during the period after the transient removal of fixation (37). Collagen II rather than aggrecan demonstrated a difference between DDH and control group, while collagen II expression was downregulated at 8 weeks after modeling, which followed a gradual elevation until 6 weeks. Conversely, aggrecan mRNA expression was downregulated until 8 weeks post-modeling. The expression levels of MMP-13 and ADAMTS-5 were significantly affected by ECM synthesis and increased at 2 weeks, which was associated with the compression loading being released. Furthermore, following re-mobilization of the hip, the expression of both proteases was downregulated. MMP-13 and ADAMTS-5 have been reported to have an important effect in early degeneration during loading stress (38). Furthermore, ADAMTS-5 expression is higher than collagen II and aggrecan during the early stages of DDH and lower in the later stages, suggesting that ADAMTS-5 is more sensitive to changes in loading stress and serves a predominant role in hip remodeling in the early stages following load removal. If complete reduction is achieved, the expression of ADAMS-5 mRNA is reversible and cartilage degeneration may be prevented, as reported by Karsdal et al (39). A study by Breckon et al (40) involving a 14-year-old patient with DDH suggested that MMP-13 was not essential for the remodeling 
A

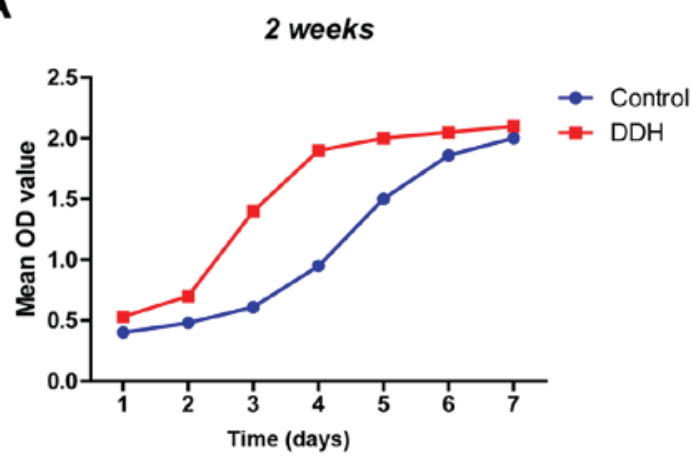

C

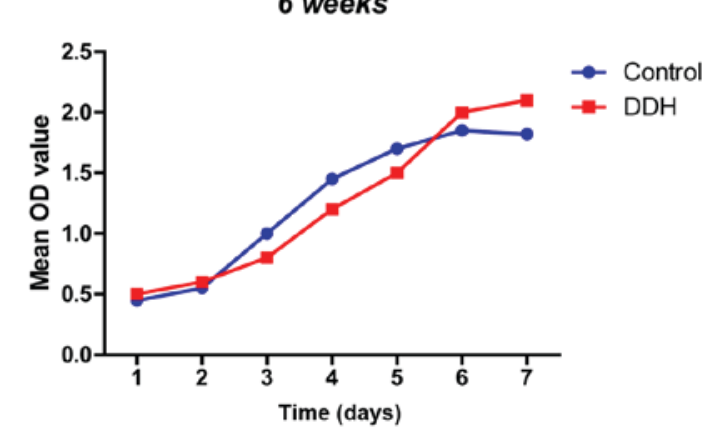

B

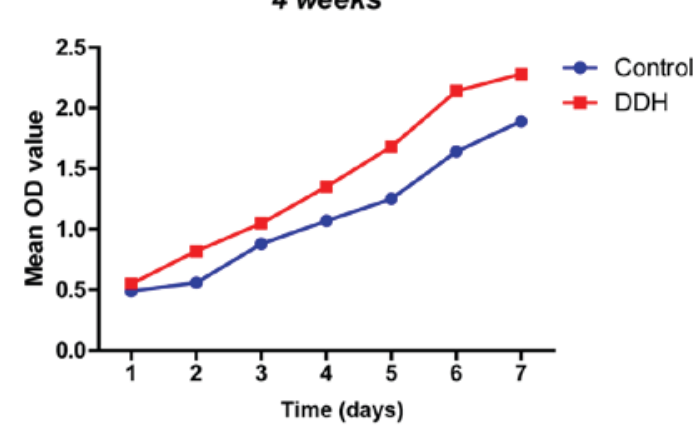

D

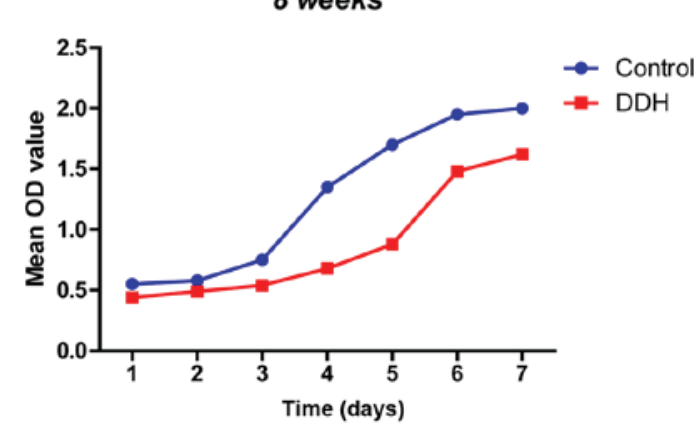

Figure 3. Cell growth from 2 to 8 weeks. (A) At 2 weeks, the experimental DDH group entered the linear growth phase, the slope of which was greater than in the control group. This was indicative of increased proliferative ability. (B) No significant differences were observed between the slopes of the cell growth curve in each group at 4 weeks. (C) The slope of the linear phase reversed at week 6 and (D) these changes were maintained until 8 weeks, with a lower slope in the experimental DDH group. OD, optical density; DDH, developmental dysplasia of the hip.

A

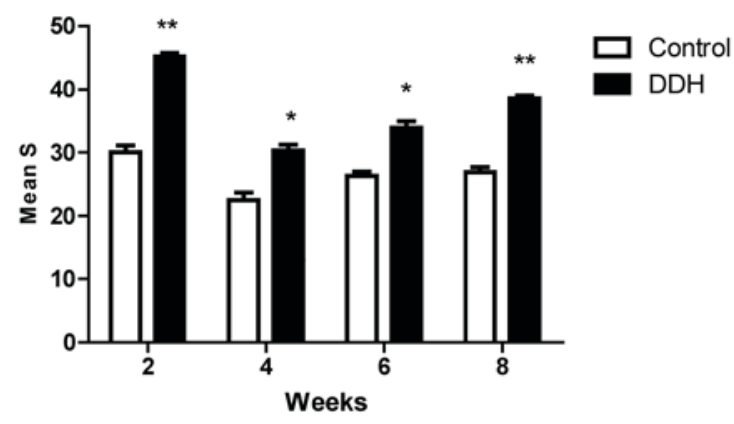

C

$S$ phase

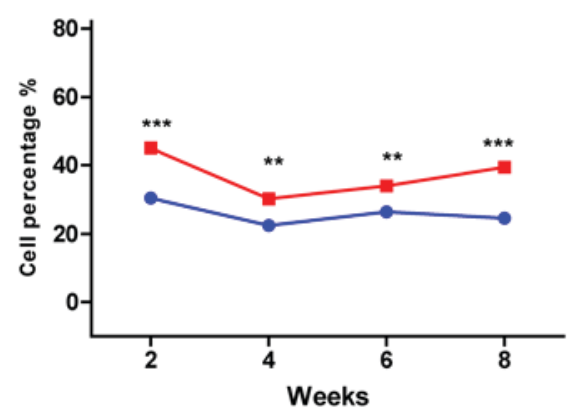

G1 phase

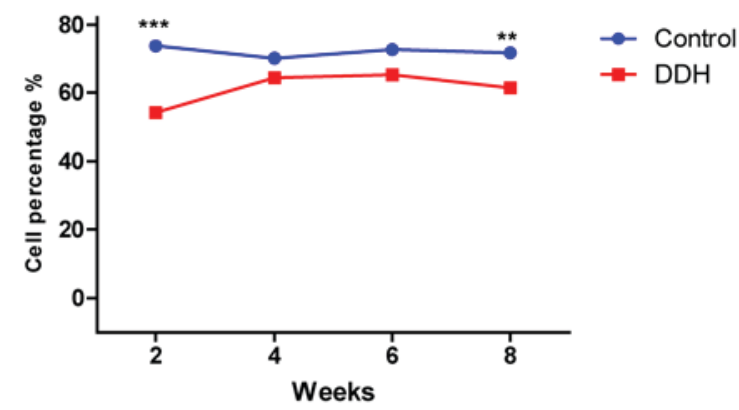

D

G2 phase

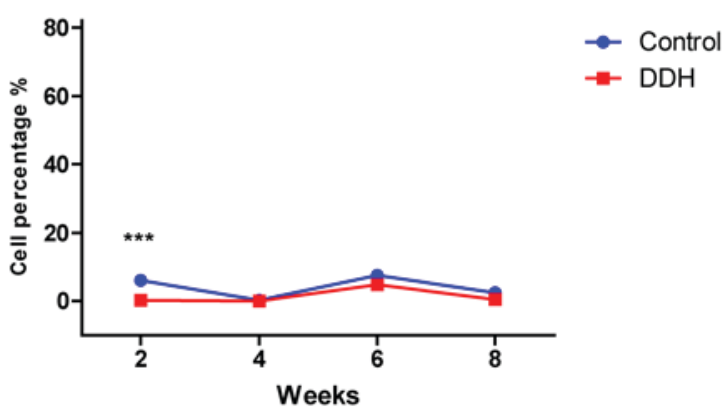

Figure 4. Number of cells in S phase increases during DDH degeneration. (A) The proportion of cells in S-phase was assessed to indicate proliferative ability. The number of cells in S-phase increased gradually after the removal of the fixation from 4 weeks. Cell cycle distribution. The number of cells in (B) G1-phase was decreased in the experimental DDH group compared with the control group, especially at 2 and 8 weeks. (C) A significant increase was presented in the number of cells in the $\mathrm{S}$ phase in the experimental DDH group from 2 to 8. Furthermore, the number of cells in the (D) G2-phase was decreased in the experimental DDH group, compared with the control group, especially at 2 weeks. ${ }^{*} \mathrm{P}<0.05,{ }^{* *} \mathrm{P}<0.01$ and ${ }^{* * * *} \mathrm{P}<0.001$ vs. control. DDH, developmental dysplasia of the hip. 
A

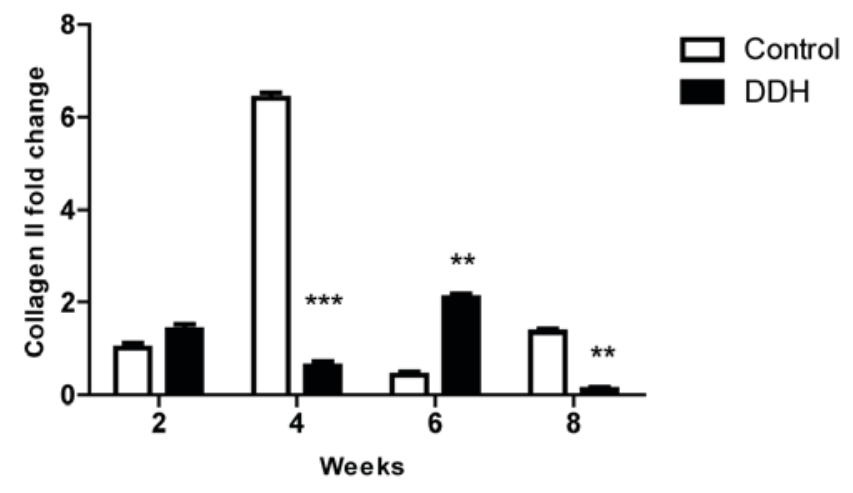

C

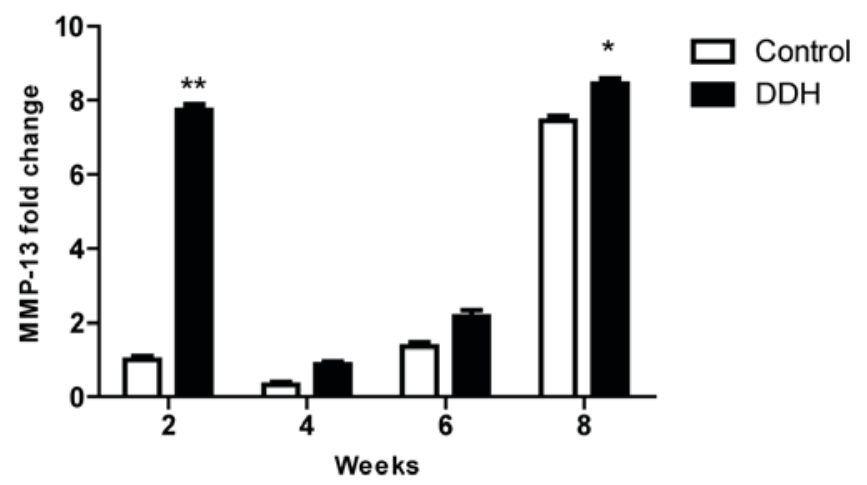

B

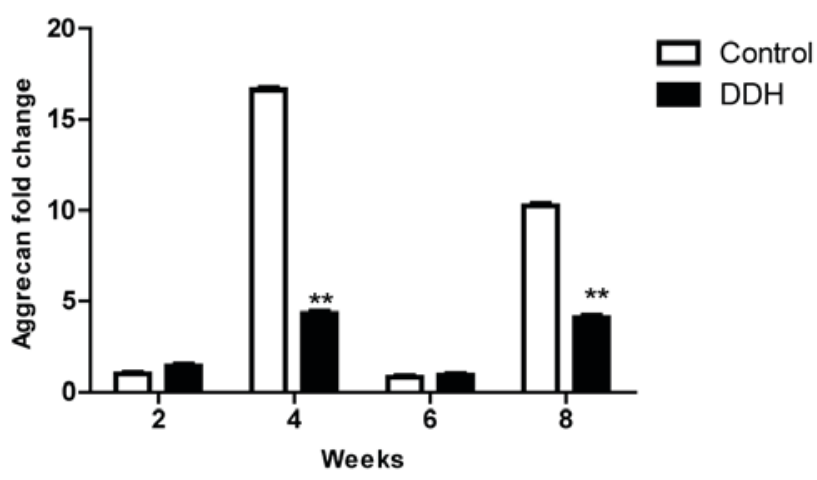

D

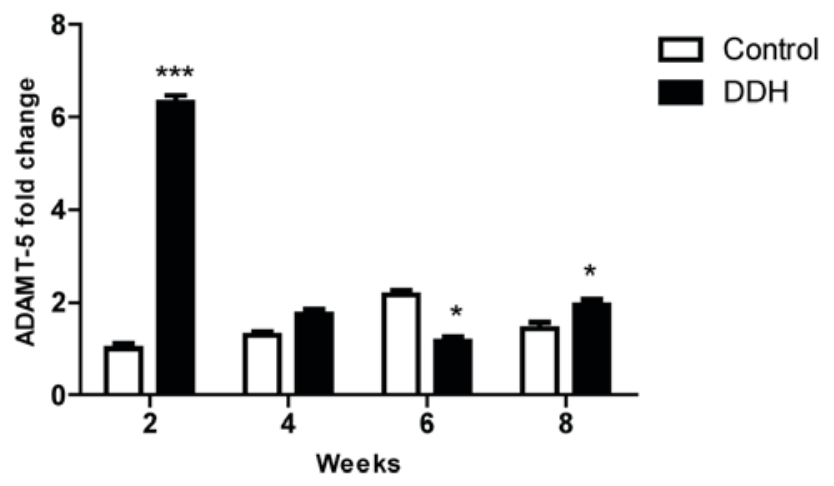

Figure 5. mRNA expression levels of collagen II, aggrecan, MMP-13 and ADAMTS-5 are varied at different time-points from 2 to 8 weeks in DDH. (A) The expression of collagen II mRNA was upregulated in the DDH group at 2 weeks after the removal of fixation, whereas it was downregulated at 4 weeks. This effect was reversed at 6 weeks followed by a further downregulation of expression at 8 weeks. (B) The expression of aggrecan mRNA was markedly different at 4 weeks compared with 8 weeks, whereas there was no great difference between 2 weeks and 6 weeks. (C) MMP-13 mRNA was overexpressed in the experimental DDH group compared with the control group from 2 to 8 weeks, and the expression in DDH was gradually upregulated during the times. (D) ADAMTS-5 mRNA expression in the experimental DDH group was markedly different compared with the control group at weeks 2,6 and 8 . "P $<0.05$, ${ }^{* *} \mathrm{P}<0.01$ and ${ }^{* * *} \mathrm{P}<0.001$ vs. control. DDH, developmental dysplasia of the hip; MMP-13, matrix metallopeptidase; ADAMTS-5, ADAM metallopeptidase with thrombospondin type 1 motif 5 .

of cartilage growth and chondrocyte proliferation. Conversely, other studies have reported that MMP-13 serves an important role in cartilage development and ECM remodeling. Previous studies have revealed that MMP-13 and ADAMTS-5 expression levels are closely associated with stress activity $(37,41,42)$, suggesting that several signal pathways could play complex roles in mediating chondrocyte metabolism in DDH cartilage. MMP-13 and ADAMTS-5 were upregulated during the early degeneration of cartilage and then downregulated sharply. This result suggests that DDH degeneration may be reversible during DDH degeneration.

It has previously been reported that collagen expression is associated with the longitudinal and transverse distribution and intensity of weight-bearing (43-47). A phenomenon known as dedifferentiation can occur during cell culture, and in the present study, early alternations in DDH cartilage at the cellular and molecular levels in vivo and in vitro resulted in no complete reduction and subluxation in consequence. This does not influence our results; although alternative findings in different tests were observed, the expression of target molecules was unaffected. Changes at the molecular level, which are the initial promoters of progressive degeneration, were not observed. Therefore, in future studies it will be interesting to focus on it whether the early degeneration occurs after the operations of DDH at molecular level and what time will be appropriate for the operation so that the degeneration could be prevented completely.

In conclusion, although complete reduction was not achieved after the removal of fixation, transient remodeling of the hip occurred over time, which was indicative of high proliferative activity in the chondrocytes as well as cell cycle progression at the early stage. However, degeneration occurred at the later stage. These results suggested that MMP-13 and ADAMTS-5 serve a dominant role not only in the remodeling phase but also in the degeneration stage.

\section{Acknowledgements}

Not applicable.

\section{Funding}

No funding was received.

\section{Availability of data and materials}

All data generated or analyzed during this study are included in this published article. 


\section{Authors' contributions}

DW designed the experiments and revised the paper. BN, RJ and LW performed the experiments and wrote the paper. BN and LW analyzed the data. BN and DW read and revised the paper.

\section{Ethics approval and consent to participate}

All methods in this study were approved by the Research Medical Ethics Committee of Fudan University. All experimental protocols were performed in accordance with the Institutional Ethics Committee of the Animal Ethical Committee of Fudan University.

\section{Patient consent for publication}

Not applicable.

\section{Competing interests}

The authors declare they have no competing interests.

\section{References}

1. Nelitz M and Reichel H: Nonsurgical treatment of developmental dysplasia of the hip. Orthopade 37: 550, 552-555, 2008 (In German)

2. Yamamoto N: Changes of the acetabular cartilage following experimental subluxation of the hip joint in rabbits. Nihon Seikeigeka Gakkai zasshi 57: 1741-1753, 1983 (In Japanese).

3. Ibrahim S: Acetabular dysplasia after treatment for developmental dysplasia of the hip. J Bone Joint Surg Br 87: 1025, 2005.

4. Kim HT, Kim JI and Yoo CI: Acetabular development after closed reduction of developmental dislocation of the hip. $\mathrm{J}$ Pediatr Orthop 20: 701-708, 2000.

5. Ma R, Ji S, Zhou Y, Liu W and Zhang L: Evolutionary regularity of acetabular dysplasia after reduction of developmental dislocation of the hip. Chin Med J (Engl) 110: 346-348, 1997.

6. Nishii T, Sugano N, Sato Y, Tanaka H, Miki H and Yoshikawa H: Three-dimensional distribution of acetabular cartilage thickness in patients with hip dysplasia: A fully automated computational analysis of MR imaging. Osteoarthritis Cartilage 12: 650-657, 2004.

7. Nishii T, Shiomi T, Tanaka H, Yamazaki Y, Murase K and Sugano N: Loaded cartilage T2 mapping in patients with hip dysplasia. Radiology 256: 955-965, 2010.

8. Sijbrandij S: Dislocation of the hip in young rats produced experimentally by prolonged extension. J Bone Joint Surg Br 47: $792-795,1965$.

9. Greenhill BJ, Hainau B, Ellis RD and el-Sayed RM: Acetabular changes in an experimental model of developmental dysplasia of the hip (DDH). J Pediatr Orthop 15: 789-793, 1995.

10. Bo N, Peng W, Xinghong P and Ma R: Early cartilage degeneration in a rat experimental model of developmental dysplasia of the hip. Connect Tissue Res 53: 513-520, 2012.

11. Casali PG and Blay JY; ESMO/CONTICANET/EUROBONET Consensus Panel of Expert: Gastrointestinal stromal tumours: ESMO clinical practice guidelines for diagnosis, treatment and follow-up. Ann Oncol 21 (Suppl 5): v98-v102, 2010.

12. Ning B, Sun J, Yuan Y, Yao J, Wang P and Ma R: Early articular cartilage degeneration in a developmental dislocation of the hip model results from activation of $\beta$-catenin. Int J Clin Exp Pathol 7: 1369-1378, 2014.

13. da Silva MA, Yamada N, Clarke NM and Roach HI: Cellular and epigenetic features of a young healthy and a young osteoarthritic cartilage compared with aged control and OA cartilage. J Orthop Res 27: 593-601, 2009.

14. Kremli MK, Alshahid AH, Khoshhal KI and Zamzam MM: The pattern of developmental dysplasia of the hip. Saudi Med J 24: $1118-1120,2003$
15. Manning WK and Bonner WM Jr: Isolation and culture of chondrocytes from human adult articular cartilage. Arthritis Rheum 10: 235-239, 1967.

16. Livak KJ and Schmittgen TD: Analysis of relative gene expression data using real-time quantitative PCR and the 2(-Delta Delta C(T)) method. Methods 25: 402-408, 2001.

17. Raab P, Lohr J and Krauspe R: Remodeling of the acetabulum after experimental hip joint dislocation-an animal experiment study of the rabbit. Z Orthop Ihre Grenzgeb 136: 519-524, 1998.

18. Ning B, Yuan Y, Yao J, Zhang S and Sun J: Analyses of outcomes of one-stage operation for treatment of late-diagnosed developmental dislocation of the hip: 864 hips followed for 3.2 to 8.9 years. BMC Musculoskelet Disord 15: 401, 2014.

19. Tsuji Y, Takeshita H, Kusuzaki K, Hirasawa Y, Ueda K and Ashihara T: Cell proliferation and differentiation of cultured chondrocytes isolated from growth plate cartilage of rat rib. Nihon Geka Hokan 64: 50-63, 1995.

20. Hunter CJ, Imler SM, Malaviya P, Nerem RM and Levenston ME: Mechanical compression alters gene expression and extracellular matrix synthesis by chondrocytes cultured in collagen I gels. Biomaterials 23: 1249-1259, 2002.

21. Garcia M and Knight MM: Cyclic loading opens hemichannels to release ATP as part of a chondrocyte mechanotransduction pathway. J Orthop Res 28: 510-515, 2010.

22. Bougault C, Paumier A, Aubert-Foucher E and Mallein-Gerin F: Molecular analysis of chondrocytes cultured in agarose in response to dynamic compression. BMC Biotechnol 8: 71, 2008.

23. De Croos JN, Dhaliwal SS, Grynpas MD, Pilliar RM and Kandel RA: Cyclic compressive mechanical stimulation induces sequential catabolic and anabolic gene changes in chondrocytes resulting in increased extracellular matrix accumulation. Matrix Biology 25: 323-331, 2006.

24. Villanueva I, Gladem SK, Kessler J and Bryant SJ: Dynamic loading stimulates chondrocyte biosynthesis when encapsulated in charged hydrogels prepared from poly (ethylene glycol) and chondroitin sulfate. Matrix Biol 29: 51-62, 2010.

25. Ando K, Imai S, Isoya E, Kubo M, Mimura T, Shioji S, Ueyama $H$ and Matsusue Y: Effect of dynamic compressive loading and its combination with a growth factor on the chondrocytic phenotype of 3-dimensional scaffold-embedded chondrocytes. Acta Orthop 80: 724-733, 2009.

26. Abusara Z, Seerattan R, Leumann A, Thompson R and Herzog W: A novel method for determining articular cartilage chondrocyte mechanics in vivo. J Biomech 44: 930-934, 2011.

27. Rolauffs B, Williams JM, Aurich M, Grodzinsky AJ, Kuettner KE and Cole AA: Proliferative remodeling of the spatial organization of human superficial chondrocytes distant from focal early osteoarthritis. Arthritis Rheum 62: 489-498, 2010.

28. Shields KJ, Beckman MJ, Bowlin GL and Wayne JS: Mechanical properties and cellular proliferation of electrospun collagen type II. Tissue Eng 10: 1510-1517, 2004.

29. Sivan SS, Wachtel E and Roughley P: Structure, function, aging and turnover of aggrecan in the intervertebral disc. Biochim Biophys Acta 1840: 3181-3189, 2014.

30. Kiani C, Chen L, Wu YJ, Yee AJ and Yang BB: Structure and function of aggrecan. Cell Res 12: 19-32, 2002.

31. Nagase $H$ and Kashiwagi M: Aggrecanases and cartilage matrix degradation. Arthritis Res Ther 5: 94-103, 2003.

32. van Meurs JB, van Lent PL, Holthuysen AE, Singer II, Bayne EK and van den Berg WB: Kinetics of aggrecanase- and metalloproteinase-induced neoepitopes in various stages of cartilage destruction in murine arthritis. Arthritis Rheum 42: 1128-1139, 1999.

33. van Lent PL, Grevers LC, Blom AB, Arntz OJ, van de Loo FA, van der Kraan P, Abdollahi-Roodsaz S, Srikrishna G, Freeze H, Sloetjes A, et al: Stimulation of chondrocyte-mediated cartilage destruction by S100A8 in experimental murine arthritis. Arthritis Rheum 58: 3776-3787, 2008.

34. Narmoneva DA, Cheung HS, Wang JY, Howell DS and Setton LA: Altered swelling behavior of femoral cartilage following joint immobilization in a canine model. J Orthop Res 20: 83-91, 2002.

35. Hagiwara Y, Ando A, Chimoto E, Saijo Y, Ohmori-Matsuda K and Itoi E: Changes of articular cartilage after immobilization in a rat knee contracture model. J Orthop Res 27: 236-242, 2009.

36. Tchetina EV, Squires G and Poole AR: Increased type II collagen degradation and very early focal cartilage degeneration is associated with upregulation of chondrocyte differentiation related genes in early human articular cartilage lesions. J Rheumatol 32: 876-886, 2005. 
37. Haapala J, Arokoski JP, Hyttinen MM, Lammi M, Tammi M, Kovanen V, Helminen HJ and Kiviranta I: Remobilization does not fully restore immobilization induced articular cartilage atrophy. Clin Orthop Relat Res: 218-229, 1999.

38. Borzi RM, Olivotto E, Pagani S, Vitellozzi R, Neri S, Battistelli M, Falcieri E, Facchini A, Flamigni F, Penzo M, et al: Matrix metalloproteinase 13 loss associated with impaired extracellular matrix remodeling disrupts chondrocyte differentiation by concerted effects on multiple regulatory factors. Arthritis Rheum 62: 2370-2381, 2010.

39. Karsdal MA, Madsen SH, Christiansen C, Henriksen K, Fosang AJ and Sondergaard BC: Cartilage degradation is fully reversible in the presence of aggrecanase but not matrix metalloproteinase activity. Arthritis Res Ther 10: R63, 2008.

40. Breckon JJ, Hembry RM, Reynolds JJ and Meikle MC: Regional and temporal changes in the synthesis of matrix metalloproteinases and TIMP-1 during development of the rabbit mandibular condyle. J Anat 184: 99-110, 1994.

41. Selvamurugan N, Jefcoat SC, Kwok S, Kowalewski R, Tamasi JA and Partridge NC: Overexpression of Runx 2 directed by the matrix metalloproteinase-13 promoter containing the AP-1 and Runx/RD/Cbfa sites alters bone remodeling in vivo. J Cell Biochem 99: 545-557, 2006.

42. Tetsunaga T, Nishida K, Furumatsu T, Naruse K, Hirohata S, Yoshida A, Saito T and Ozaki T: Regulation of mechanical stress-induced MMP-13 and ADAMTS-5 expression by RUNX-2 transcriptional factor in SW1353 chondrocyte-like cells. Osteoarthritis Cartilage 19: 222-232, 2011.
43. Aigner T, Stoss H, Weseloh G, Zeiler G and von der Mark K: Activation of collagen type II expression in osteoarthritic and rheumatoid cartilage. Virchows Arch B Cell Pathol Incl Mol Pathol 62: 337-345, 1992.

44. Aigner T, Bertling W, Stöss H, Weseloh G and von der Mark K: Independent expression of fibril-forming collagens I, II, and III in chondrocytes of human osteoarthritic cartilage. J Clin Invest 91: 829-837, 1993.

45. Aigner T, Vornehm SI, Zeiler G, Dudhia J, von der Mark K and Bayliss MT: Suppression of cartilage matrix gene expression in upper zone chondrocytes of osteoarthritic cartilage. Arthritis Rheum 40: 562-569, 1997.

46. Hotta H, Yamada H, Takaishi H, Abe T, Morioka H, Kikuchi T, Fujikawa $\mathrm{K}$ and Toyama Y: Type II collagen synthesis in the articular cartilage of a rabbit model of osteoarthritis: Expression of type II collagen C-propeptide and mRNA especially during early-stage osteoarthritis. J Orthop Sci 10: 595-607, 2005.

47. Park K, Min BH, Han DK and Hasty K: Quantitative analysis of temporal and spatial variations of chondrocyte behavior in engineered cartilage during long-term culture. Ann Biomed Eng 35: 419-428, 2007.

This work is licensed under a Creative Commons Attribution-NonCommercial-NoDerivatives 4.0 International (CC BY-NC-ND 4.0) License. 